\title{
In vivo validation of near-infrared light transillumination for interproximal dentin caries detection
}

\author{
Jan Kühnisch $^{1}$ • Friederike Söchtig ${ }^{1}$ Vinay Pitchika ${ }^{1}$. Rüdiger Laubender ${ }^{2}$. \\ Klaus W. Neuhaus ${ }^{3} \cdot$ Adrian Lussi $^{3} \cdot$ Reinhard Hickel $^{1}$
}

Received: 4 June 2014 / Accepted: 3 August 2015 /Published online: 15 September 2015

(C) Springer-Verlag Berlin Heidelberg 2015

\begin{abstract}
Objectives The aims of this clinical study were to investigate the diagnostic accuracy of near-infrared light transillumination (NILT) as a novel X-ray-free method for proximal dentin caries detection and to compare this method to established diagnostic methods.

Materials and methods A total of 127 interproximal dentin caries lesions without any cavity within visible dentin in posterior teeth from 85 consecutively selected patients were included. Visual and radiographic diagnoses and laser fluorescence measurements were available. NILT images were obtained, and a dentin lesion was predicted if a demineralisation involved the enamel-dentin junction (NILT-EDJ) or a shadow in dentin was detectable (NILT-dentin). Included lesions were opened and validated (reference standard). The statistical analyses included descriptive analyses and calculations of sensitivity, specificity and Az values.

Results The diagnostic accuracy with respect to the reference standard was $1.6 \%$ for visual inspection, $66.7 \%$ for laser fluorescence, $96.1 \%$ for digital radiography, $29.1 \%$ for NILT-dentin and $99.2 \%$ for NILT-EDJ. Bitewings (Az 0.984 ) and NILT-EDJ (Az 0.992) performed equally.
\end{abstract}

Jan Kühnisch

jkuehn@dent.med.uni-muenchen.de

1 Department of Conservative Dentistry and Periodontology, Ludwig-Maximilians-University of Munich, 80336 Munich, Germany

2 Institute for Medical Informatics, Biometry and Epidemiology, Ludwig-Maximilians-University of Munich, Munich, Germany

3 Department of Preventive, Restorative, and Pediatric Dentistry, University of Bern, Bern, Switzerland
Conclusion Given the lack of true negatives in the study, the diagnostic accuracy of NILT achieved the same level as bitewings for the detection of proximal dentin caries.

Clinical relevance This study might indicate that NILT could reduce the usage of bitewings.

Keywords Interproximal caries $\cdot$ Dentin caries $\cdot$ Caries detection $\cdot$ Caries diagnostics $\cdot$ Near-infrared light transillumination $\cdot$ DIAGNOcam

\section{Introduction}

X-ray-free, near-infrared light transillumination (NILT) is a photo-optical method for caries detection and diagnosis in posterior teeth, and it was first described in 1995 [1]. This method is a further development of the digital imaging fibreoptic transillumination method (DiFOTI), which has gained clinical attention in recent years [2-5]. The DiFOTI method uses visible light, whereas the NILT uses invisible long-wave light. The main advantage of longer wavelengths is the decreased scattering, which allows near-infrared light to penetrate objects more deeply [6]. As a result, near-infrared light could be transmitted easily through solid dental enamel and could achieve good contrast between a caries lesion and the surrounding sound hard tissue [7]. Furthermore, the use of light at $830 \mathrm{~nm}$ offers improved image contrast over the visible range, although there is less contrast compared with longer wavelengths [8]. Another major modification of the NILT is that the illuminating light is shone around the alveolar process but not directly into the interproximal space $[9,10]$. Both modifications have considerably improved the imaging quality of interproximal sites from the occlusal aspect. This effect appears to be remarkable, as no ionising radiation is used to visualise the interproximal caries process. 
The clinical potential of NILT for interproximal caries detection and diagnosis was shown in one feasibility study, which included 33 caries lesions from 18 test subjects [9]. This study demonstrated that NILT has considerable potential for the imaging of early decay; however, the authors stated that they used a camera prototype that was not convenient to use for in vivo imaging. With respect to the need to improve clinical handling and to reduce equipment costs, the camera design was consequently modified. The result of these technological modifications was a novel NILT camera system (DIAGNOcam, KaVo, Biberach, Germany) that was introduced on the European dental market in autumn of 2012. This camera was designed according to the above-mentioned photo-optical principles and uses an illuminating wavelength of $780 \mathrm{~nm}$. With this system, it appears to be possible to capture different stages of interproximal enamel and dentin caries lesions (Fig. 1). Dentin caries lesions could be detected in cases in which the demineralisation of the enamel involved the enamel-dentin junction (NILT-EDJ, Fig. 1). Deep dentin caries lesions were found to be linked with a dark dentin shadow (NILT-dentin, Fig. 1). Due to the limited clinical data [9], the need to validate this novel diagnostic device is urgent. This in vivo study aimed to estimate the diagnostic accuracy of the newly introduced NILT camera for detection of interproximal dentin caries in comparison to the validated caries depth after opening the lesion. Further, established and validated diagnostic methods, e.g. bitewing radiography, laser fluorescence (LF) measurements and visual inspection, were considered. It was hypothesised that this new photo-optical diagnostic device has the same ability to detect proximal dentin caries lesions compared to other diagnostic methods. Because operative treatment of sound teeth or enamel caries lesions in humans for validation reasons is not possible for ethical reasons, such proximal surfaces were not considered.

Subjects and methods This prospectively designed clinical study on diagnostic accuracy was approved by the Ethics Committee of the Medical Faculty of the Ludwig-MaximiliansUniversity of Munich (project number 013-12). The reporting of this investigation followed the recommendations of the Standard for Reporting of Diagnostic Accuracy (STARD) steering committee [11]. Prior to the beginning of this study, a power calculation was performed. Expecting a sensitivity or specificity of $95 \%$ for NILT, a minimum of 98 interproximal lesions should be included. For this assumption, the $95 \%$ confidence interval was calculated to be 88.1 to 107.9.

Participants The patient recruitment was performed in the Department of Conservative Dentistry in Munich (J.K., F.S. and others). The patient-related inclusion criteria were a classification of status 1 (healthy patient), according to the American Society of Anaesthesiologists, fully erupted permanent
Fig. 1 NILT images of enamel and dentin interproximal caries lesions. $\mathbf{a}$ and $\mathbf{b}$ show enamel caries lesions with different configurations. c presents a lesion that is associated with complete demineralisation of the enamel and involvement of the EDJ, with no signs of a dentin caries lesion (NILT-EDJ). In addition to these characteristics, the interproximal caries lesion in $\mathbf{d}$ shows less translucent dentin, which could be detected as a dark area (NILTdentin)
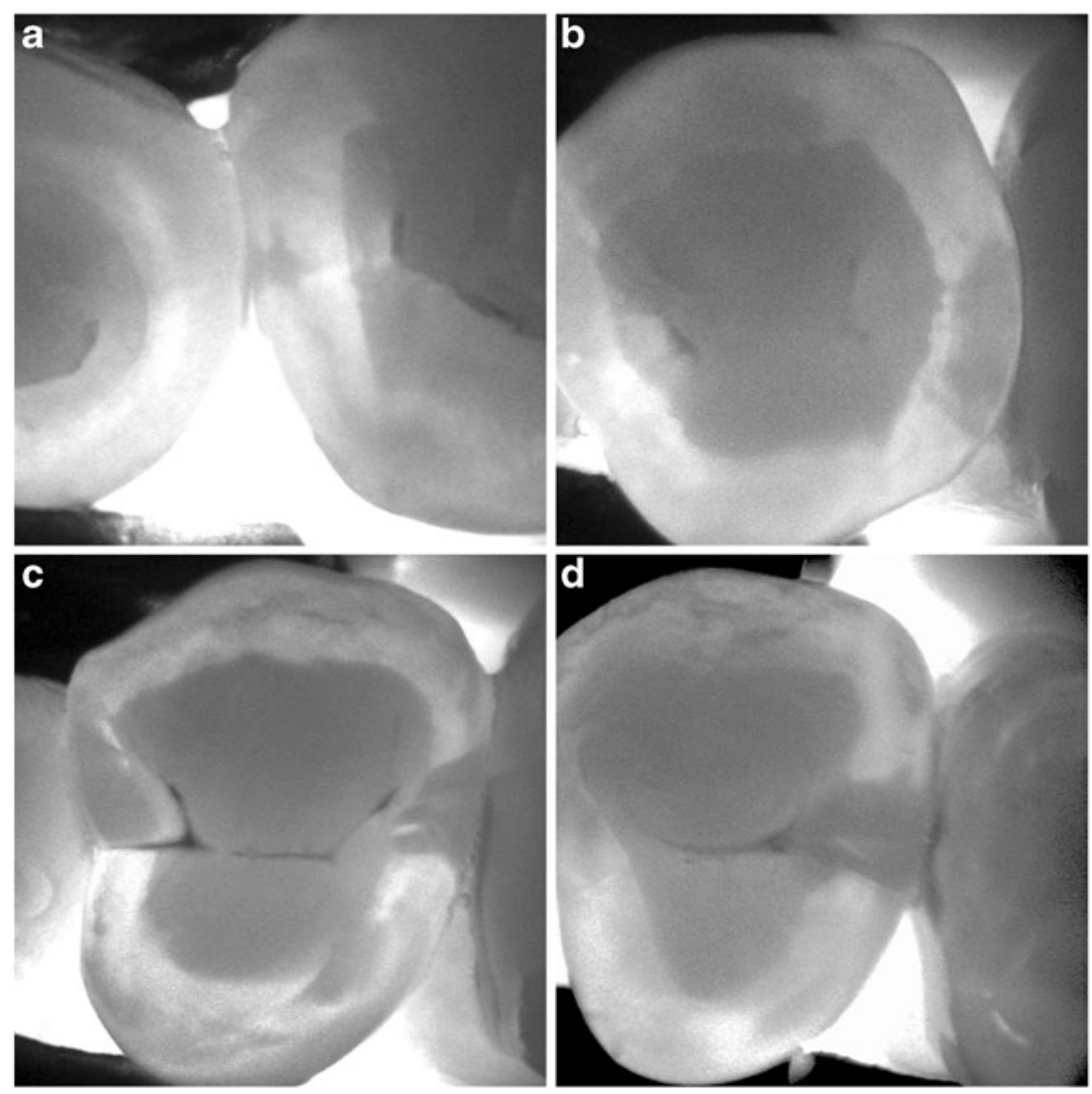
dentition and a minimum age of 12 years. Considering the tooth-related inclusion criteria, a minimum of one symptomless, interproximal dentin caries lesion without any visible cavity in the posterior teeth was necessary. Teeth with visible proximal dentin cavitations [12-15] were not considered in this study. Interproximal surfaces with restorations, secondary caries, residual caries and orthodontic bands were excluded from this study. To specify the diagnosis "interproximal dentin caries" bitewing radiographs were primarily required. Therefore, each patient was asked to recall whether bitewing radiography had been administered over the last 4 months. If the answer was positive, these images were requested, included and evaluated. In the case of a negative response and present clinical indicators that warranted bitewing radiography (e.g. non-cavitated carious lesions at smooth and/or proximal surfaces, existing caries risk or activity), it was prescribed. In such cases, an intraoral X-ray dental machine with a 203-mm tube (Heliodent DS, Sirona, Bensheim, Germany) including an X-ray field limitation $(30 \times 40 \mathrm{~mm})$ was used with a charge-coupled device (CCD) sensor (Intraoral II, sensor size $30.7 \times 40.7 \mathrm{~mm}$, Sirona, Bensheim, Germany). The exposure time was $0.06 \mathrm{~s}$ at a cathode voltage of $60 \mathrm{kV}$ and $7 \mathrm{~mA}$ of amperage. A sensor-holding device (XPP-DS Digital Sensor Holders for Sirona, Dentsply Rinn, Elgin, IL, USA) was used at all times. All the digital radiographs were primarily analysed by the recruiting dentists (F.S. and J.K.). In case of the diagnosis of an interproximal dentin caries lesion (D3-4 lesion), all relevant treatment strategies were discussed with the patient. When needing operative dental care, the patient was asked to take part in this diagnostic study. Following the informed consent which was obtained from each participant, a separate appointment for validation and restoration was made.

Study population The potential participants who did not meet the inclusion criteria were excluded $(N=18)$; mostly no informed consent was given or the patients missed the appointment for validation (Fig. 2). The consecutive series of participants included 85 patients ( 38 male/47 female) with a mean age of 25.0 years (standard deviation, 6.4) and showed a moderate caries experience (5.5 DMFT/10.9 DMFS). Finally, 127 untreated posterior teeth (first premolars $=28$, second premolars $=53$, first permanent molars $=38$, and second permanent molars $=7$ ) with varying degrees of interproximal carious dentin lesions and without any visible cavity were included. To minimise the influence of any single participant, a maximum of two lesions per patient were allowed. The patients were treated from May 2012 to January 2013.

Clinical validation At this separate appointment, each selected site was carefully re-investigated clinically [12-15], and the bitewing radiographs were re-considered before validation. The indication for validation was made only when an unmistakable dentin caries lesion on digital radiographs (D3/ 4) and/or an unambiguous presence of dentin involvement (NILT-dentin) on NILT images was present. Non-cavitated caries lesions, equivocal radiolucencies nearby the EDJ on bitewings or translucencies which reached the EDJ punctual on NILT images were not validated and excluded from this study to avoid any overtreatment. Thus, the decision for operative dental care was predominately based on combined clinical and radiographical assessment. All the diagnostic test procedures, validation methods and restorative measures were performed immediately after professional tooth cleaning, using standard conditions and a professional dental unit with an operation light, plane dental mirror and compressed air, operated by a trained dentist (F.S.). The independent analysis of all NILT images and bitewing radiographs as well as the determination of the reference standard was performed blindly after the clinical study phase with a minimum interval of 2 weeks.

Visual caries scoring Each interproximal site was carefully re-examined visually without tooth separation. The lesion severity (e.g. first signs, established lesion, localised enamel breakdown) was scored according to the International Caries Detection and Assessment System (ICDAS) [12] (www.icdas. org) and the Universal Visual Scoring System (UniViSS) [13, 14] (www.univiss.net).

Laser fluorescence examination LF measurements of the interproximal sites were performed by a dentist (F.S.) using an LF with a tapered tip (DIAGNOdent Pen/2190, KaVo, Biberach, Germany). The identical device was used for all the examinations. Calibration of the probe with the ceramic standard was performed according to the manufacturer's instructions. Then, the device was individually adjusted to the autofluorescence of the tooth at a healthy site after brief airdrying. Next, the tapered tip was introduced into the interproximal space as close as possible to the contact point [16]. The maximum LF reading (0-99) was recorded. Interproximal dentin caries were associated with values $\geq 16$ [17].

Digital radiography All the digital radiographs were primarily analysed by the recruiting dentists (F.S. and J.K.). All the images were evaluated independently from all the other diagnostic findings in a darkened room using the available systemrelated analysis software (version 2.53, Sirona, Bensheim, Germany) with the option of adjusting the brightness and contrast by a dentist (F.S.). For each surface, the corresponding categorical diagnosis (D-score) was made as follows: D0 - sound surface, D1 - caries restricted to the outer one half of the enamel, D2 - caries restricted to the inner one half of the enamel and D3/4 - caries restricted to the outer/inner one half of the dentin [18]. All the radiographic decisions were re-assessed before the appointment for validation in collaboration with an experienced examiner (J.K.) to achieve a diagnostic consensus. For 
Fig. 2 Flow diagram of the progress through this diagnostic trial

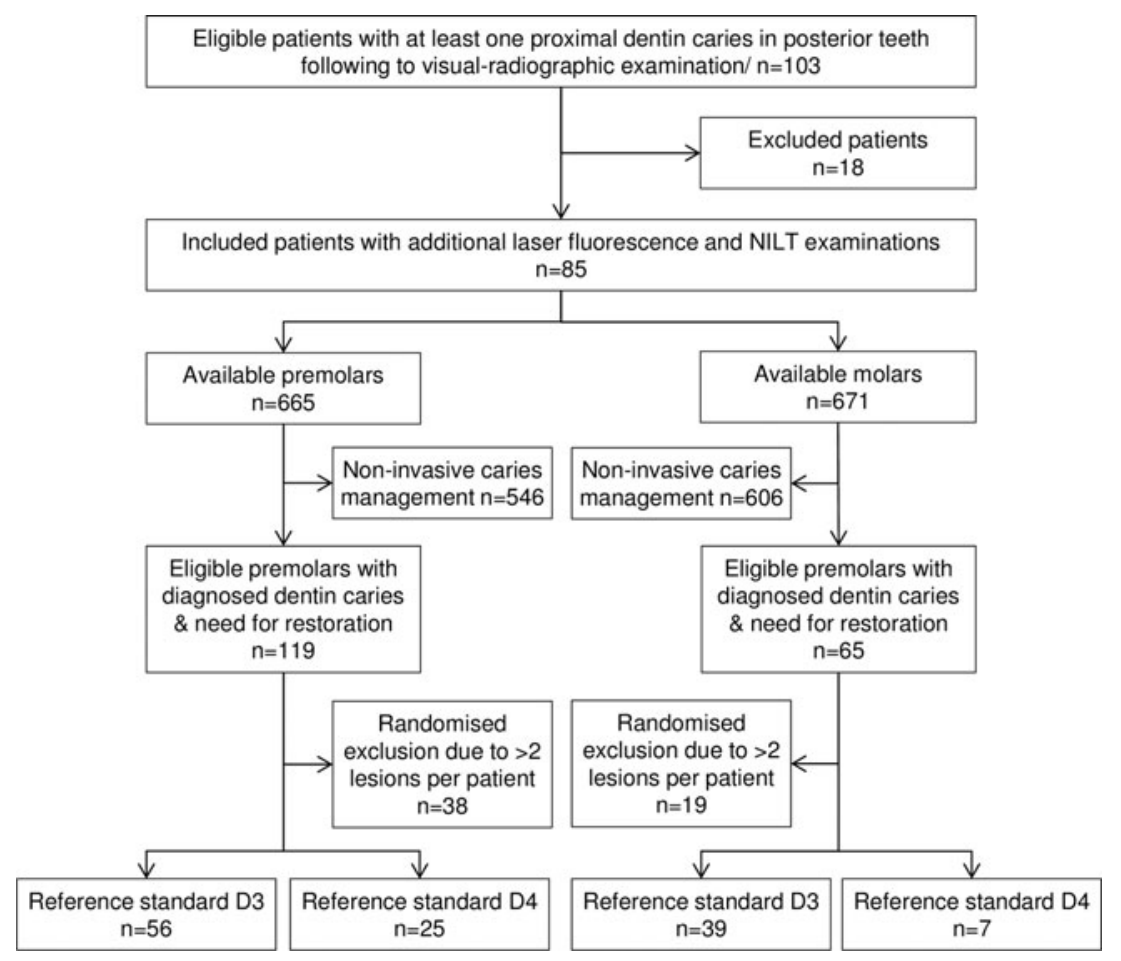

cases in which the examiners reached different conclusions, they re-assessed the corresponding radiographs and discussed their points until a consensus was reached.

NILT After air-drying, each of the included interproximal sites was examined with the NILT camera perpendicularly centred over the area of interest. The light source on the dental unit was switched off while the images were obtained to prevent any light interference. The images were captured and stored with KID software (KaVo Integrated Desktop/version 2.4.1.6374, KaVo, Biberach, Germany). Following the clinical phase, the NILT images were first analysed by one dentist (F.S.) independently from the other diagnostic findings. On the basis of the given examples for caries lesions in Fig. 1, the enamel (Fig. 1a, b) and dentin caries lesions (Fig. 1c, d) were categorised. A dentin lesion was predicted as follows: first, if the enamel was completely demineralised and the enamel-dentin junction (EDJ) was involved (NILT-EDJ, Fig. 1c), and second, if demineralisation in the enamel penetrated the EDJ and a less translucent shadow in the dentin was detectable (NILT-dentin, Fig. 1d). Additionally, the NILT images were also re-assessed at least 2 weeks later under the supervision of an experienced dentist (J.K.) to achieve a consensus diagnosis. When the examiners reached different conclusions, they re-assessed the images, discussed their points and modified the decision accordingly.

\section{Caries extension and characteristics (reference standard)}

The first validation step was careful opening of the interproximal dentin lesion with a conical diamond burr to confirm whether a carious dentin lesion was present beneath the EDJ. The lesion characteristics of hardness, moisture and colouration were recorded. Following this evaluation, the caries process was completely excavated with a round carbide burr in the periphery of the dentin. The inner soft carious dentin was removed with a self-limiting polymer burr (P1, Komet, Lemgo, Germany). After bevelling the enamel margins (SonicSys, KaVo, Biberach, Germany), a silicon impression (Optosil and Xantopren, Heraeus Dental, Hanau, Germany) of the final cavity was obtained. The cavity was restored with an adhesive restoration (Syntac classic, Vivadent, Schaan, Lichtenstein; SONICfill, KaVo, Biberach, Germany; SonicFILL, West Collins, Orange, CA, USA).

Following the clinical phase of this study, the quantification of the extent of the caries was done (Fig. 3). This required the stone model (Cerec stone BC, Sirona Dental Systems, Bensheim, Germany), which was made from the silicon impression. The model was then X-rayed, and the resulting Xray (jpeg file) was converted into a transparent image (gif file). In the next step, this image was superimposed exactly on the in vivo bitewing (jpg file) using image-editing software (Photo-Paint Essentials 4, Corel Corp, Ottawa, Canada), and the cavity floor was marked. To calculate the D-score [18] and the CE index [14], the distance between the EDJ and the marked cavity floor was set relative to the overall dentin thickness between the EDJ and the pulp. The CE index was expressed as percental dentin involvement for each lesion. For statistical analysis, all values were categorised as following: caries in the outer quarter of dentin (CE index $<25 \%$ ), caries in the middle quarters of dentin ( $>25-50$ and $>50-75 \%$ ) and caries in the 
Fig. 3 The standardised workflow for the validation of the extension of caries included the replication of the caries depth clinically. Following caries excavation and the bevelling of the cavity margins, a silicon impression was obtained. In the dental laboratory, a stone model was created, and the surplus material was trimmed away. The model was then X-rayed with the alignment set-up for bitewing radiographs. In the next step, the in vivo bitewing (jpg file) and the corresponding in vitro $\mathrm{X}$-ray from the stone model (transparent gif file) were exactly superimposed using image-editing software. Next, the cavity floor was marked, the in vitro radiograph was removed and the resulting image was separately stored so that the distance between the cavity floor and the EDJ or pulp could be measured

\section{In vitro replication of the cavity and determination of the caries depth in relation to the pulp}

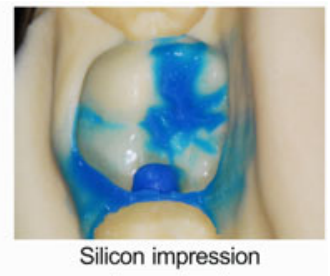

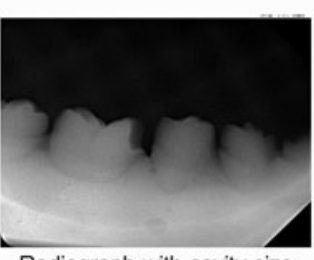

Radiograph with cavity size
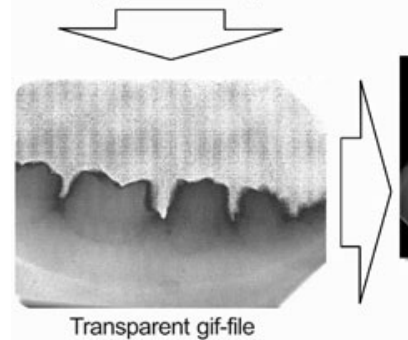

Transparent gif-file

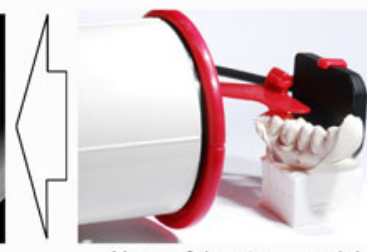

$\mathrm{X}$-ray of the stone model

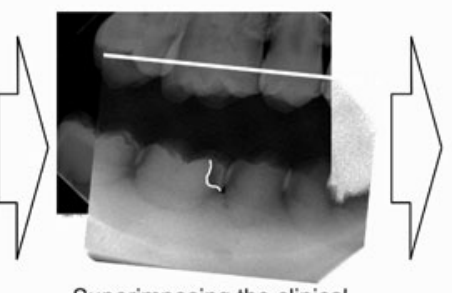

Superimposing the clinical bitewing with the radiograph from the stone model

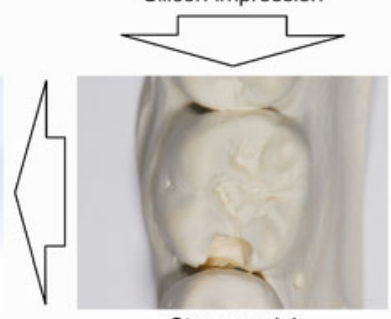

Stone model

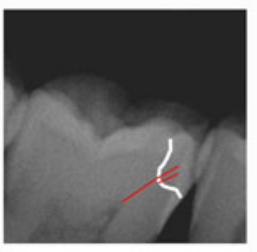

Removing of the gif-file and metric analysis inner quarter of dentin near the pulp $(<75-100 \%)$. The correctness of the CE index was counterchecked at least 2 weeks later under the supervision of an experienced dentist (F.S. and J.K.) to achieve a diagnosis by consensus. Different findings were discussed, resulting in the correction of the previously made decision according to the new diagnosis. The reference standard was determined independently from all other diagnostic findings.

Calibration Prior to the study, a 2-day theoretical and practical calibration training, which focused on the clinical standardisation of all the test methods used in the study and the reference standard, was conducted by an experienced dentist (J.K.) to instruct the main examiner (F.S.). The theoretical training exercise provided information regarding the study design, indices, diagnostic principles and standardised validation procedure (Fig. 3). This training session was followed by a clinical training course, during which the examiner validated, under supervision, ten carious dentin lesions, which were not included in the study. The weighted kappa values for the intra- and inter-examiner reproducibility were good to excellent (NILT 0.910 (F.S./intra), 0.850 (J.K. intra) and 0.810 (inter); bitewing 0.808 (F.S./intra), 0.808 (J.K. intra) and 0.744 (inter)).

Statistical analysis The data were analysed using SPSS software, version 20.0 (IBM Corp., Armonk, NY, USA), and R software (http://www.r-project.org). The overall diagnostic accuracy $($ accuracy $=(\mathrm{TN}+\mathrm{TP}) /(\mathrm{TN}+\mathrm{TP}+\mathrm{FN}+\mathrm{FP}))$ was determined by calculating the number of true positives (TP), false positives (FP), true negatives (TN) and false positives (FP) for each method. Further, sensitivity (SE), specificity (SP), positive and negative predictive values (PPV and NPV, respectively), as well as the areas under the ROC curves (Az), were computed [19]. Whereas the calculation of SE, SP, PPV, and NPV required dichotomised data, the categorical (visual examination, digital radiography, NILT) or continuous data (LF) were used to plot the ROC curves.

\section{Results}

Out of 127 lesions, 94 lesions were rated as D3 lesions and 33 lesions were rated as D4 lesions according to the reference standard. None of the included sites were free of caries or were found in the enamel only. Table 1 presents the cross-tabulation for main diagnostic scores in relation to the validated caries depth. The characteristics after opening up the caries lesion beneath the EDJ, with regard to the extent of the validated caries, are provided in Table 2 . The results from crosstabulations and diagnostic accuracy parameters are shown in Table 3. The best diagnostic performance for the prediction of interproximal dentin caries lesions, with regard to the reference standard, was documented for digital radiography and NILT-EDJ; the corresponding $\mathrm{Az}$ values of 0.984 and 0.992 indicated excellent accuracy. 
Table 1 Correlation between the diagnostic findings for each method and the validated caries extension in dentin

\begin{tabular}{|c|c|c|c|c|c|}
\hline \multirow[t]{3}{*}{ Diagnostics } & \multicolumn{4}{|c|}{ Percental/categorial caries extension in dentin (reference standard) } & \multirow{3}{*}{ Total } \\
\hline & \multicolumn{2}{|l|}{ D3 } & \multicolumn{2}{|l|}{ D4 } & \\
\hline & $<25 \%$ & $>25-50 \%$ & $>50-75 \%$ & $>75 \%$ & \\
\hline \multicolumn{6}{|l|}{ Visual examination } \\
\hline No finding & 23 & 38 & 15 & 6 & 82 \\
\hline First signs & 7 & 13 & 10 & 1 & 31 \\
\hline Established lesion & 2 & 10 & - & - & 12 \\
\hline Microcavity & 1 & 1 & - & - & 2 \\
\hline Total & 33 & 62 & 25 & 7 & 127 \\
\hline \multicolumn{6}{|l|}{ Digital radiography } \\
\hline D2 & 3 & 2 & - & - & 5 \\
\hline D3 & 29 & 56 & 10 & - & 95 \\
\hline D4 & 1 & 4 & 15 & 7 & 27 \\
\hline Total & 33 & 62 & 25 & 7 & 127 \\
\hline \multicolumn{6}{|l|}{ LF } \\
\hline$\geq 16$ & 10 & 25 & 6 & 1 & 42 \\
\hline$>16$ & 22 & 37 & 19 & 6 & 84 \\
\hline Missing & 1 & - & - & - & 1 \\
\hline Total & 33 & 62 & 25 & 7 & 127 \\
\hline \multicolumn{6}{|l|}{ NILT } \\
\hline NILT-EDJ & 31 & 49 & 9 & 1 & 90 \\
\hline NILT-dentin & 2 & 13 & 16 & 6 & 37 \\
\hline Total & 33 & 62 & 25 & 7 & 127 \\
\hline & 94 & & 33 & & \\
\hline
\end{tabular}

The percentage of lesions that were categorised as NILT-dentin increased with the caries depth (CE of 0-25\%, two detectable cases versus 31 non-detectable cases; CE of 25-50\%, 13/49; CE of 50-75\%, 16/9; CE of $75-100 \%, 6 / 1)$. No adverse events or other side effects of the test method were recorded.
Table 2 Characterisation of the dentin beneath the EDJ during validation in relation to the dentin caries extension

\begin{tabular}{|c|c|c|c|c|c|}
\hline \multirow[t]{3}{*}{ Lesion characteristics beneath the EDJ } & \multicolumn{4}{|c|}{ Percental/categorial caries extension in dentin (reference standard) } & \multirow{3}{*}{ Total } \\
\hline & \multicolumn{2}{|l|}{ D3 } & \multicolumn{2}{|l|}{ D4 } & \\
\hline & $<25 \%$ & $>25-50 \%$ & $>50-75 \%$ & $>75 \%$ & \\
\hline \multicolumn{6}{|l|}{ Hardness } \\
\hline Soft & 9 & 22 & 19 & 6 & 56 \\
\hline Leathery & 12 & 32 & 6 & 1 & 51 \\
\hline Hard & 11 & 8 & 0 & 0 & 20 \\
\hline Total & 33 & 62 & 25 & 7 & 127 \\
\hline \multicolumn{6}{|l|}{ Moisture } \\
\hline Wet & 6 & 27 & 19 & 6 & 58 \\
\hline Intermediate & 17 & 27 & 6 & 1 & 51 \\
\hline Dry & 9 & 8 & 0 & 0 & 18 \\
\hline Total & 33 & 62 & 25 & 7 & 127 \\
\hline \multicolumn{6}{|l|}{ Colouration } \\
\hline None/light brown & 20 & 33 & 19 & 7 & 80 \\
\hline Dark brown/black & 12 & 29 & 6 & 0 & 47 \\
\hline Total & 33 & 62 & 25 & 7 & 127 \\
\hline & 94 & & 33 & & \\
\hline
\end{tabular}


Table 3 Performance of the diagnostic methods used in this study compared with the validated extension of caries for dentin caries detection on the interproximal surfaces of permanent posterior teeth

\begin{tabular}{|c|c|c|c|c|c|}
\hline & Visual examination $^{\mathrm{a}}$ & $\mathrm{LF}^{\mathrm{b}}$ & NILT-dentin & NILT-EDJ & Digital radiography \\
\hline \multicolumn{6}{|l|}{ Results from cross-tabulations } \\
\hline True positives/TP $(\mathrm{N})$ & 2 & 84 & 37 & 126 & 122 \\
\hline True negatives/TN (N) & 0 & 0 & 0 & 0 & 0 \\
\hline False positives/FP (N) & 0 & 0 & 0 & 0 & 0 \\
\hline False negatives/FN (N) & 125 & 42 & 90 & 1 & 5 \\
\hline \multicolumn{6}{|l|}{ Diagnostic accuracy } \\
\hline Accuracy $(\%)$ & 1.6 & 66.7 & 29.1 & 99.2 & 96.1 \\
\hline Sensitivity/SE (\%) & 1.6 & 66.7 & 29.1 & 99.2 & 96.1 \\
\hline Sensitivity $(95 \% \mathrm{CI})$ & $0.4-5.6$ & $58.1-74.3$ & $21.9-37.6$ & $95.7-99.9$ & $91.1-98.3$ \\
\hline Specificity/SP (\%) & $\mathrm{nc}^{\mathrm{c}}$ & $\mathrm{nc}^{\mathrm{c}}$ & $\mathrm{nc}^{\mathrm{c}}$ & $\mathrm{nc}^{\mathrm{c}}$ & $\mathrm{nc}^{\mathrm{c}}$ \\
\hline Specificity $(95 \%$ CI) & $\mathrm{nc}^{\mathrm{c}}$ & $\mathrm{nc}^{\mathrm{c}}$ & $\mathrm{nc}^{\mathrm{c}}$ & $\mathrm{nc}^{\mathrm{c}}$ & $\mathrm{nc}^{\mathrm{c}}$ \\
\hline Positive predictive value (\%) & 100.0 & 100.0 & 100.0 & 100.0 & 100.0 \\
\hline Negative predictive value (\%) & $\mathrm{nc}^{\mathrm{c}}$ & $\mathrm{nc}^{\mathrm{c}}$ & $\mathrm{nc}^{\mathrm{c}}$ & $\mathrm{nc}^{\mathrm{c}}$ & $\mathrm{nc}^{\mathrm{c}}$ \\
\hline Positive post-test probability & 1 & 1 & 1 & 1 & 1 \\
\hline Negative post-test probability & 1 & 1 & 1 & 1 & 1 \\
\hline Area under the ROC curve/Az & 0.680 & 0.820 & 0.648 & 0.992 & 0.984 \\
\hline
\end{tabular}

${ }^{\text {a }}$ Visual detectable localised breakdowns of enamel or distinct cavitation were used as the cut-off value for dentin caries detection

${ }^{\mathrm{b}}$ LF values $\geq 16$ were used to predict dentin caries. One case was excluded because of heterogeneous measurements

${ }^{\mathrm{c}}$ Not calculable because of the non-validation of sound surfaces and enamel caries lesions

\section{Discussion}

Before interpreting the clinical importance of our results from this clinical diagnostic study, several methodological aspects should be discussed critically. With regard to this in vivo study, great care was taken to avoid any overtreatment [20]. After the patient recruitment, the primary diagnosis of dentin caries was carefully counterchecked by the study team. Given that NILT was a new diagnostic method that had not been supported by clinical experience when we began our study, the findings from visual examination and the caries extension on bitewing radiography were predominately used as reference for the decision-making process. With this careful planning for treatment and the fact that digital radiography tended to have higher specificities [20], we clearly avoided operative interventions on remineralisable or infiltrable interproximal enamel caries lesions (Table 3). In consequence, this means that only dentin lesions were included in this study. Due to this unbalanced case distribution, it is worth mentioning that diagnostic accuracy needs to be interpreted cautiously so far. This important issue is linked with an "absolute SnNout" (negative results from highly sensitive tests can rule a diagnosis out/ sensitive, negative, out $=$ SnNOut), which could be observed when the selection criteria resulted in any spectrum bias [21, 22]. Because our study followed basic ethical demands and did not validate sound sites or enamel caries lesions, high sensitivity was registered, and a negative result thus ruled out the diagnosis. Because of missing negative test results, the specificity values were not calculable (Table 3 ). This selection bias, which is linked to the inclusion of notable proximal dentinal caries lesions detected primarily on bitewing radiographs and missing negative controls, limits the generalisability of our results but fulfils the basic ethical requirements. The selection bias might have an effect on the evaluation of NILT images because the probability of diagnosing dentin caries was high. This finding implies that the core aim of a validation study to distinguish between sound enamel or dentin lesions or to measure the extent of the lesions with a novel device could only be addressed under in vitro conditions, which requires the development of an appropriate laboratory model. Our attempts to establish an appropriate in vitro set-up have failed to transilluminate the teeth sufficiently because the photo-optical properties of the different embedding materials are not comparable with periodontal anatomy (e.g. the oral mucosa, alveolar bone, periodontal ligament, pulp and blood). An alternative procedure would be to re-assess the sound sites and enamel caries lesions after a distinct time period to exclude false-negative decisions and to construct negative controls [17]. However, this procedure requires a longitudinal study design and could not exclude further uncertainties because of the lack of histological or bioptical validation. A composite reference standard that combines several validation 
methods (radiography, visual inspection) appears to be legitimate; however, it is not widely accepted in dentistry [23].

Regardless of the limitations, our study provided important clinical information. Our hypothesis was that the ability of this novel photo-optical diagnostic device to detect proximal dentin caries lesions would be identical to that of other diagnostic methods; however, this hypothesis must be rejected. Whereas interproximal dentin caries lesions could be detected perfectly with digital radiography and NILT-EDJ relative to the validated caries extent (Table 3), all the other methods performed worse. Compared to NILT-EDJ, our findings were as follows: (1) the visual examination performance was inferior; (2) the LF resulted in false-negative findings, which resulted in reduced diagnostic accuracy; and (3) the NILT-dentin was frequently linked with deep dentin caries lesions and could not detect dentin lesions in the outer one half of the dentin (Table 3). Although the diagnostic performance of visual inspection, laser fluorescence and bitewing radiography corresponded to earlier reports [17, 24-26], the finding that NILT performed similarly to digital radiography appears to be remarkable, and it might indicate that this method could reduce the usage of bitewing radiographs. One of the main advantages of bitewing radiographs lies in their visualisation of the caries extent relative to the EDJ and the pulp. Although the EDJ is clearly detectable on the NILT images, the pulp could not be visualised with the present NILT method. This missing information requires further analyses that aim to identify possible indicators that could be used to predict the quantitative extent of caries into the dentin instead of using categories only. This would be helpful for the clinician to differentiate between D3 and D4 caries and to estimate the caries depth in relation to the pulp.

Another result was that deep interproximal dentin caries lesions were frequently found in association with a less translucent shadow in the dentin on the NILT images (NILT-dentin). This finding explains the less-than-optimal accuracy for dentin caries detection because the majority of the lesions in the outer part of the dentin would obviously be overlooked. This diagnostic marker could therefore be understood as a "safety net" for dental practitioners by indicating an immediate need for operative dental treatment. This assumption is supported by the frequencies of the parameters that are linked with active caries lesions, e.g. soft, wet and with light brown discolouration. These parameters were more frequently registered as the depth of caries increased (Table 2).

A unique feature of our study was the methodology used to determine the actual extent of caries clinically. Given the impossibility of verifying the reference point of the pulp under in vivo conditions, we aimed to eliminate this general drawback. Using the key features to replicate the cavity and superimpose the clinical bitewing radiograph onto the radiograph from the stone model, the extent of the in vivo caries could be determined more accurately than by using simple estimation. Nevertheless, future investigations should analyse the precision of this procedure. Additionally, the relevant information regarding the lesion characteristics was sampled, and it was shown that the majority of the indicators for an active caries lesion, e.g. soft, wet and less discoloured dentin, are present more frequently in deep dentin caries lesions (Table 2). Nevertheless, we found that several active dentin caries lesions progressed only into the outer one quarter of the dentin (Table 2). Considering the clinical difficulty of performing a reliable visual(-tactile) examination of the proximal sites with or without tooth separation as well as the limited availability of diagnostic methods or devices to predict lesion activity, caries extension is a major indicator in distinguishing between preventive and operative dental care. This assessment could be performed reliably with bitewing radiographs because the extent of caries is visualised relative to the EDJ and the pulp. Other diagnostic methods, including NILT, must be defined as alternative measures to predict the extent of caries or the need for operative dental care. Other exclusive features of this study are the power calculation, the use of a self-limiting instrument to objectify caries excavation and the independent consensus diagnoses on all of the diagnostic images. Because numerous images were discussed extensively, a non-biased investigation of the intra- and interexaminer reproducibility was not possible and should therefore be included in future trials.

\section{Conclusions}

The extensively discussed selection bias influenced our study results; however, this type of bias cannot be excluded from any in vivo diagnostic study for ethical reasons. In addition, because of the unavailability of an appropriate in vitro model for the investigation of the NILT, a clinical study was the only opportunity for estimating the usefulness of this new diagnostic method for the dental practitioner to date. Our in vivo diagnostic study, which fulfilled level $1 \mathrm{c}$ according to the Oxford Centre for Evidence-Based Medicine [27], revealed similar diagnostic accuracy for interproximal dentin caries detection when using digital radiography and NILT-EDJ. This encouraging finding indicates that future clinical and laboratory work should focus on the investigation of the diagnostic accuracy of these methods on occlusal surfaces, on the assessment of the intra- and inter-examiner reproducibility and on the development of treatment-related recommendations for NILT. With respect to the strength of NILT for visualising interproximal enamel caries lesions (Fig. 1a, b), the potential for early primary caries detection should also be considered.

Conflict of interest The clinical work was supported by KaVo (Biberach, Germany). The authors declare that they have no conflicts of interest with regard to the authorship and/or publication of this article. Further, the publication of the study results was not hindered by any requests from the financial supporter. 


\section{References}

1. Fried D, Glena RE, Featherstone JD, Seka W (1995) Nature of light scattering in dental enamel and dentin at visible and near-infrared wavelengths. Appl Opt 34:1278-1285

2. Young DA, Featherstone JD (2005) Digital imaging fiber-optic trans-illumination, F-speed radiographic film and depth of approximal lesions. J Am Dent Assoc 136:1682-1687

3. Young DA (2002) New caries detection technologies and modern caries management: merging the strategies. Gen Dent 50:320-331

4. Wenzel A, Verdonschot EH, Truin GJ, Konig KG (1992) Accuracy of visual inspection, fiber-optic transillumination, and various radiographic image modalities for the detection of occlusal caries in extracted non-cavitated teeth. J Dent Res 71:1934-1937

5. Schneiderman A, Elbaum M, Shultz T, Keem S, Greenebaum M, Driller J (1997) Assessment of dental caries with Digital Imaging Fiber-Optic TransIllumination (DIFOTI): in vitro study. Caries Res 31:103-110

6. Hall A, Girkin JM (2004) A review of potential new diagnostic modalities for caries lesions. J Dent Res 83 (Spec Issue No C): C89-C94

7. Jones R, Huynh G, Jones G, Fried D (2003) Near-infrared transillumination at $1310-\mathrm{nm}$ for the imaging of early dental decay. Opt Express 11:2259-2265

8. Jones GC, Jones RS, Fried D (2004) Transillumination of interproximal caries lesions with 830-nm light. Proc SPIE 5313, Lasers in Dentistry X, 17 (May 28, 2004)

9. Staninec M, Lee C, Darling CL, Fried D (2010) In vivo near-IR imaging of approximal dental decay at 1,310 nm. Lasers Surg Med 42:292-298

10. Chung S, Fried D, Staninec M, Darling CL (2011) Near infrared imaging of teeth at wavelengths between 1200 and $1600 \mathrm{~nm}$. Proc Soc Photo Opt Instrum Eng. 2011 Jan 1;7884. pii: 78840X

11. Bossuyt PM, Reitsma JB, Bruns DE, Gatsonis CA, Glasziou PP, Irwig LM, Lijmer JG, Moher D, Rennie D, de Vet HCW, for the STARD Group (2003). Towards complete and accurate reporting of studies of diagnostic accuracy: The STARD Initiative. BMJ 326: $41-44$

12. Pitts NB (2009) Detection, assessment, diagnosis and monitoring of caries. Monographs in Oral Science, Vol. 21. Basel: S. Karger AG

13. Kühnisch J, Goddon I, Berger S, Senkel H, Bücher K, Oehme T, Hickel R, Heinrich-Weltzien R (2009) Development, methodology and potential of the new Universal Visual Scoring System
(UniViSS) for caries detection and diagnosis. Int J Environ Res Public Health 6:2500-2509

14. Kühnisch J, Bücher K, Henschel V, Albrecht A, Garcia-Godoy F, Mansmann U, Hickel R, Heinrich-Weltzien R (2011) Diagnostic performance of the universal visual scoring system (UniViSS) on occlusal surfaces. Clin Oral Investig 15:215-223

15. WHO (1997) Oral health surveys. Basic methods. 4th edition, World Health Organization, Geneva

16. Lussi A, Hellwig E (2006) Performance of a new laser fluorescence device for the detection of occlusal caries in vitro. J Dent 34:467-471

17. Huth KC, Lussi A, Gygax M, Thum M, Crispin A, Paschos E, Hickel R, Neuhaus KW (2010) In vivo performance of a laser fluorescence device for the approximal detection of caries in permanent molars. J Dent 38:1019-1026

18. Marthaler TM (1966) A standardized system of recording dental conditions. Helv Odontol Acta 10:1-18

19. Matthews DE, Farewell VT (2007) Using and understanding medical statistics, 4th edn. S. Karger GmbH, Freiburg

20. Baelum V (2010) What is an appropriate caries diagnosis. Acta Odontol Scand 68:65-79

21. Kühnisch J, Ifland S, Tranæus S, Heinrich-Weltzien R (2009) Comparison of visual inspection and different radiographic methods for dentine caries detection on occlusal surfaces. Dentomaxillofac Radiol 38:452-457

22. Pewsner D, Battaglia M, Minder C, Marx A, Bucher HC, Egger M (2004) Ruling a diagnosis in or out with "SpPIn" and "SnNOut": a note of caution. BMJ 329:209-213

23. Hegedus EJ, Stern B (2009) Beyond SpPIN and SnNOUT: considerations with dichotomous tests during assessment of diagnostic accuracy. J Man Manip Ther 17:E1-E5

24. Rutjes AW1, Reitsma JB, Coomarasamy A, Khan KS, Bossuyt PM. Evaluation of diagnostic tests when there is no gold standard. A review of methods. Health Technol Assess. 2007 11(50):iii, ix-51

25. Lussi A, Hibst R, Paulus R (2004) DIAGNOdent: an optical method for caries detection. J Dent Res 83(Spec Iss C) C80-83

26. Bader JD, Shugars DA (2004) A systematic review of the performance of a laser fluorescence device for detecting caries. J Am Dent Assoc 135:1413-1426

27. Oxford centre for evidence-based medicine (2009). Levels of evidence. URL: www.cebm.net/index.aspx?o=4590 (accessed June 3th 2013) 